\title{
A curious relation between the flat cosmological model and the elliptic integral of the first kind (Research Note)
}

\author{
A. Mészáros ${ }^{1}$ and J. Řípa ${ }^{2}$ \\ ${ }^{1}$ Charles University in Prague, Faculty of Mathematics and Physics, Astronomical Institute, V Holešovičkách 2, 18000 Prague 8 , \\ Czech Republic \\ e-mail: meszaros@cesnet.cz \\ 2 Institute of Basic Science, Natural Sciences Campus, Sungkyunkwan University, Engineering Building 2, 2066 Seobu-ro, \\ Jangan-gu, Suwon, 440-746 Gyeonggi-do, Korea \\ e-mail: ripa.jakub@gmail.com
}

Received 14 June 2013 / Accepted 16 June 2013

\section{ABSTRACT}

Context. The dependence of the luminosity distance on the redshift has a key importance in the cosmology. This dependence can well be given by standard functions for the zero cosmological constant.

Aims. The purpose of this article is to present a similar relation also for the non-zero cosmological constant, if the universe is spatially flat.

Methods. A definite integral was used.

Results. The integration ends in the elliptic integral of the first kind.

Conclusions. The result shows that no numerical integration is needed for the non-zero cosmological constant if the universe is spatially flat.

Key words. cosmology: theory

\section{Introduction}

The dependence of the luminosity distance $d_{\mathrm{L}}(z)$ on the redshift $z$ is a key formula in cosmology (Carroll et al. 1992). It is given by three independent cosmological parameters: by two omegaparameters $\Omega_{\mathrm{M}}, \Omega_{\Lambda}$, and by the Hubble constant $H_{0}$. Its relation to the so-called proper-motion distance is given by $d_{\mathrm{PM}}(z)(1+$ $z)=d_{\mathrm{L}}(z)$ (Weinberg 1972).

One has (Carroll et al. 1992)

$$
\begin{aligned}
& d_{\mathrm{PM}}(z)=\frac{c}{H_{0} \sqrt{\left|\Omega_{k}\right|}} \\
& \times \operatorname{sinn}\left\{\sqrt{\left|\Omega_{k}\right|} \int_{0}^{z} \frac{\mathrm{d} z^{\prime}}{\sqrt{\left(1+z^{\prime}\right)^{2}\left(1+\Omega_{\mathrm{M}} z^{\prime}\right)-z^{\prime}\left(2+z^{\prime}\right) \Omega_{\Lambda}}}\right\}
\end{aligned}
$$

In this equation $c$ is the speed of light in vacuum, and it holds $\Omega_{k}+\Omega_{\mathrm{M}}+\Omega_{\Lambda}=1$. The notation "sinn" means the standard function sinh for $\Omega_{k}>0$, and $\sin$ for $\Omega_{k}<0$, respectively. If $\Omega_{k}=0$, then one simply has an integration:

$$
\frac{H_{0} d_{\mathrm{PM}}(z)}{c}=\int_{0}^{z} \frac{\mathrm{d} z^{\prime}}{\sqrt{\left(1+z^{\prime}\right)^{2}\left(1+\Omega_{\mathrm{M}} z^{\prime}\right)-z^{\prime}\left(2+z^{\prime}\right) \Omega_{\Lambda}}} .
$$

In addition, from the physical point of view, in both equations it must be $\Omega_{\mathrm{M}}>0$. (The case $\Omega_{\mathrm{M}}=0$ can serve as a limit, but $\Omega_{\mathrm{M}}<0$ is fully unphysical.) On the other hand, $\Omega_{\Lambda}$ can have both signs, but the observations of the past two decades strongly disfavour negative values (see, e.g., Perrett et al. 2012, and references therein).
In the special case of $\Omega_{\Lambda}=0$, the integral in Eq. (1) can be given by the so-called Mattig-formula (Mattig 1958) for any $\Omega_{\mathrm{M}}>0$. The formula can then easily be used in cosmological applications (see, e.g., Mészáros 2002). For $\Omega_{\Lambda} \neq 0$ the integral in Eqs. (1), (2) is usually solved numerically ${ }^{1}$.

In this note we show that the integral on the right-hand side of Eq. (2) can be solved analytically also for $\Omega_{\Lambda} \neq 0$.

\section{Integration}

We rewrite the right-hand side of Eq.(2) into the form

$\frac{H_{0} d_{\mathrm{PM}}\left(z_{0}\right)}{c}=I\left(\Omega_{\mathrm{M}}, z_{0}\right)=\int_{0}^{z_{0}} \frac{\mathrm{d} z}{\sqrt{(1+z)^{3} \Omega_{\mathrm{M}}+\Omega_{\Lambda}}}$,

where $z \geq 0, \Omega_{M}>0$ and $\Omega_{M}+\Omega_{\Lambda}=1$. Introducing the substitution $x=(1+z)^{-1}$, we obtain

$I\left(\Omega_{\mathrm{M}}, x_{0}\right)=\int_{x_{0}}^{1} \frac{\mathrm{d} x}{\sqrt{x} \sqrt{\Omega_{\mathrm{M}}+\Omega_{\Lambda} x^{3}}}$,

where $0<x_{0}=\left(1+z_{0}\right)^{-1} \leq 1$.

In this section we consider only the case $\Omega_{\mathrm{M}}<1$, i.e. $\Omega_{\Lambda}=$ $1-\Omega_{M}>0$. Introducing a further substitution $y=\left(\Omega_{\Lambda} / \Omega_{M}\right)^{1 / 3} x$, we obtain

$I\left(\Omega_{\mathrm{M}}, x_{0}\right)=\frac{1}{\Omega_{\mathrm{M}}^{1 / 3} \Omega_{\Lambda}^{1 / 6}} \int_{y_{1}}^{y_{2}} \frac{\mathrm{d} y}{\sqrt{y} \sqrt{1+y^{3}}}$,

1 See, cf.,

http://www. astro.ucla.edu/ wright/cosmolog.htm 
where $y_{1}=\left(\Omega_{\Lambda} / \Omega_{\mathrm{M}}\right)^{1 / 3} x_{0}$ and $y_{2}=\left(\Omega_{\Lambda} / \Omega_{\mathrm{M}}\right)^{1 / 3}$. The two limits are non-negative with $y_{1} \leq y_{2}$.

We aim to find the primitive function for

$$
\int \frac{\mathrm{d} y}{\sqrt{y} \sqrt{1+y^{3}}}
$$

where $y \geq 0$. The following substitution helps:

$$
\alpha=\arccos \left(\frac{1+(1-\sqrt{3}) y}{1+(1+\sqrt{3}) y}\right) .
$$

There is a one-to-one correspondence between $\alpha$ and $y$. For $y=0$ one has $\alpha=0$, and for $y \rightarrow \infty$ one has $\alpha_{\infty} \rightarrow \arccos ((1-$ $\sqrt{3}) /(1+\sqrt{3})$ ), i.e. $\alpha_{\infty}=105.54^{\circ}$. If $y$ increases in the interval $[0, \infty), \alpha$ is also increasing in the interval $\left[0, \alpha_{\infty}\right)$. Hence, this substitution is well-defined. Conversely, one obtains

$y=\frac{1-\cos \alpha}{(\sqrt{3}-1)+(\sqrt{3}+1) \cos \alpha}$

and

$\mathrm{d} y=\frac{2 \sqrt{3} \sin \alpha \mathrm{d} \alpha}{[\sqrt{3}(1+\cos \alpha)-(1-\cos \alpha)]^{2}}$.

Using these two formulas, we curiously obtain

$$
\frac{\mathrm{d} y}{\sqrt{y} \sqrt{1+y^{3}}}=\frac{\mathrm{d} \alpha}{3^{1 / 4} \sqrt{1-\frac{2+\sqrt{3}}{4} \sin ^{2} \alpha}} .
$$

The right-hand side of Eq. (10) is the function in the elliptic integral of the first kind (Gradshteyn et al. 2007) with

$$
m=\frac{2+\sqrt{3}}{4}
$$

where $0<m<1$, as it should be in an elliptic integral.

To calculate the definite integral

$\int_{y_{1}}^{y_{2}} \frac{\mathrm{d} y}{\sqrt{y} \sqrt{1+y^{3}}}$

in Eq. (5) for non-negative $y_{1} \leq y_{2}$, one can write

$$
\int_{y_{1}}^{y_{2}} \frac{\mathrm{d} y}{\sqrt{y} \sqrt{1+y^{3}}}=\int_{0}^{y_{2}} \frac{\mathrm{d} y}{\sqrt{y} \sqrt{1+y^{3}}}-\int_{0}^{y_{1}} \frac{\mathrm{d} y}{\sqrt{y} \sqrt{1+y^{3}}} \cdot
$$

After this one should use the formula with $\alpha$ from Eq. (10) and determine the integration limits in variable $\alpha$. The substitution from Eq. (7) gives for $y=0$ the value $\alpha=0$. This means that - using $\alpha$ - the lower limits in both definite integrals are zeros. The upper limits from $y_{1}$ and $y_{2}$, respectively, are also calculable analytically and unambiguously from Eq. (7) via the arccos function. One obtains $\alpha_{1}$ and $\alpha_{2}$, respectively, as upper limits in the integrals. It must be $\alpha_{2}>\alpha_{1}$. One should only clarify that, of course, if it were $\alpha_{2}$ from the interval $\left(\pi / 2, \alpha_{\infty}\right)$, then the first elliptic integral itself should be given by a sum of two integrals: in one integral the limits should be 0 and $\pi / 2$, and in the second one the limits should be $\left(\pi-\alpha_{2}\right)$ and $\pi / 2$. Both integrals must give positive values. If it were also $\alpha_{1}>\pi / 2$, then one should proceed similarly in the second integral, too. In any case, the integral $I\left(\Omega_{\mathrm{M}}, x_{0}\right)$ is easily obtainable from standard elliptic integrals of the first kind.

\section{Remarks}

The integral in Eq. (5) is presented by Gradshteyn et al. (2007) (formula 3.166.22). Moreover, Carroll et al. (1992) reported that the integral of Eq. (2) can also be solved analytically. Paál et al. (1992) described similar efforts. But, on the other hand, we found nothing in the literature about the non-numerical integration of Eq. (2) using the elliptic integrals. Therefore, the substitution given by Eqs. (7), (8) is new and original.

For the sake of completeness it should still be added that integral of Eq. (4) can be solved also for $\Omega_{\Lambda}<0$ and $\Omega_{\mathrm{M}}>1$. One obtains (up to a constant) the formula $\mathrm{d} y / \sqrt{y\left(1-y^{3}\right)}$, which is also integrable (see the formula 3.166.23 of Gradshteyn et al. 2007).

\section{Conclusion}

We have proven that the integral on the right-hand side of Eq. (2) can also be solved analytically using the elliptic integral of the first kind.

Acknowledgements. We wish to thank M. Kř́žžek for the useful discussions and comments on the manuscript. This study was supported by the OTKA Grant K77795, by the Grant Agency of the Czech Republic Grant P209/10/0734, by the Research Program MSM0021620860 of the Ministry of Education of the Czech Republic, and by Creative Research Initiatives (RCMST) of MEST/NRF.

\section{References}

Carroll, S. M., Press, W. H., \& Turner, E. L. 1992, ARA\&A, 30, 499

Gradshteyn, I. S., Ryzhik, I. M., Jeffrey, A., \& Zwillinger, D. 2007, Table of Integrals, Series, and Products (Elsevier Academic Press)

Mattig, W. 1958, Astron. Nachr., 284, 109

Mészáros, A. 2002, ApJ, 580, 12

Paál, G., Horváth, I., \& Lukács, B. 1992, Ap\&SS, 191, 107

Perrett, K., Sullivan, M., Conley, A., et al. 2012, AJ, 144, 59

Weinberg, S. 1972, Gravitation and Cosmology: Principles and Applications of the General Theory of Relativity (Wiley) 